Journal of Clinical and Nursing Research

Research Article

\title{
The Clinical Experience of Prof. Zhang Xiaofeng in the Treatment of Small Follicular, Ovulatory Infertility with Traditional Chinese Medicine
}

\author{
Huan $\mathrm{Li}^{1}$, Xiaofeng Zhang ${ }^{2 *}$, Zhe $\mathrm{Li}^{1}$ \\ The First Clinical Medical College of Traditional Chinese Medicine, Shaanxi \\ ${ }^{1}$ University of Traditional Chinese Medicine, Xianyang 712046, China; \\ ${ }^{2}$ Xi' an Hospital of Traditional Chinese Medicine, Xi' an 710021, China
}

\begin{abstract}
With the popularization of clinical ultrasound technology, small follicular ovulation infertility is gradually being recognized in clinical practice. Low follicle ovulation refers to the phenomenon that the average value of the three diameter lines of the dominant follicle is less than $14 \mathrm{~mm}-18 \mathrm{~mm}$ under continuous ultrasound monitoring. The dominant follicle ovulates before it develops to maturity, which is a kind of ovulation disorder and one of the leading causes of unexplained infertility $^{[1]}$, accounting for $25 \%-30 \%$ of the total number of patients with infertility ${ }^{[2]}$. At present, Western medicine mostly uses ovulation, stimulating drugs to treat this disease. Still, due to the clinical result of "high ovulation but low pregnancy", there are certain obstacles in treatment. The purpose of this article is to discuss Zhang Xiaofeng's understanding of TCM of this disease. The tutor believes that the location of the disease is in the kidney, and the mechanism of the disease is kidney yin deficiency and blood heat. The treatment principle is sequential therapy, nourishing yin and clearing heat, taking into account the protection of the spleen and stomach, and the therapeutic effect is perfect.
\end{abstract}

Keywords: Small follicle ovulation; Kidney yin deficiency and blood heat type; Infertility; Xiaofeng Zhang

Publication date: September, 2020

Publication online: 30 September, 2020

*Corresponding author: Xiaofeng Zhang, zhangxf2211@163.com

Prof. Zhang Xiaofeng is a famous traditional Chinese medicine practitioner in Shaanxi province and has been practicing medicine for more than 30 years, specializing in the diagnosis and treatment of gynecological diseases such as polycystic ovary syndrome, endometriosis, menstrual disorder, infertility, recurrent miscarriage, etc. $\mathrm{He}$ is good at combining traditional Chinese medicine treatment with modern medical auxiliary examination methods to treat gynecological diseases and has sound clinical effects in dispatching prescriptions. Under the tutor's instruction, I learned from him by collecting cases, arranging notes and consulting literature. The following is a summary of my mentor's experience in treating small follicular ovulation type infertility.

\section{Mechanisms of small follicular, ovulatory infertility}

Professor Zhang Xiaofeng believes that the pathogenesis of small follicle ovulation lies in the kidneys in TCM, and the main problem is the post-menstrual period, where the kidneys are deficient in yin and blood heat. The late menstrual period is equivalent to the follicular phase in Western medicine, during which yin grows, and yang disappears, and Tianqi is the primary substance that promotes follicle growth. At this time, Yin deficiency generates internal heat for a long time, which disturbs the uterine and cellular veins, resulting in the premature discharge of the immature follicle, which may cause small follicle ovulation. As the immature follicle is deficient in kidney yin, the quality of the egg is abnormal, and the egg cannot develop into a fertilized 
egg even if yin and yang fight each other.

\section{Treatment principles.}

\subsection{Sequential therapy, nourishing Yin and clearing heat}

Professor Zhang Xiaofeng attaches importance to Chinese medicine artificial cycle sequential therapy when treating small follicle ovulation. During menstruation, because of the function of the uterus of the cell, we should use prescriptions that promote menstruation and blood circulation to clear the uterus, such as Chuan Xiong, Yi Mu Cao, Chuan Niu Zhi, Zhi Zhi Hao, Danshen, Zhi Haifeng, Safflower, Peach Seed, Liu Fei Nu, Wang Bu Liu Xing, etc. The treatment focuses on the post-menstrual period, which is equivalent to the follicular phase in Western medicine. The therapy focuses on the late menstrual period, which is equal to the follicular phase in Western medicine, where sufficient Kidney Yin can promote the maturation of the dominant follicle. In the case of Kidney-Yin deficiency, symptoms such as internal heat, night sweats, tinnitus, soreness and weakness of the waist and knees can be seen. The follicles are nourished with moistening drugs that feed Kidney-Yin and evident deficiency of heat to sustain their growth. During the late menstrual period, the activity of Qi and Blood is relatively still, and the thicker the taste, the more Yin. The Su Wen-Yin and Yang Ying Xiang Da Guan say "A deficiency of form warms the Qi, a deficiency of essence complements the taste." During this period, Chinese herbal medicines such as Angelica Sinensis, fried white peony, Rehmannia, mulberry tree, custard seed, chaste seed, Mo dry lotus, fried yam, wine essence, tortoise board and turtle shell are applied. If you are deficient in yin and heat, the treatment should be supplemented with a small amount of heatclearing medicine to prevent heat from disturbing the cellular pulse and disturbing the premature discharge of immature follicles. Although the inter-menstrual period is a time when yin turns to yang, and the activities of qi and blood are more intense, to comply with the trend of turning yin to yang, warming yang and invigorating blood should be used to promote the smooth discharge of the dominant follicle. However, if the patient is deficient in yin and has internal heat, internal heat can encourage yang energy. If yang is in full bloom for a prolonged period, internal temperature will be contained and transformed into warmth, and if yang is active, yang will rise, all of which can promote the early discharge of immature follicles. This phase of the medication should not be too agitated, the application of blood circulation and dissipation of the dissipation of symptoms class of drugs to rupture the follicle, such as angelica, Chuanxiong, Dang Shen, fried white atractylodes, Salvia divinorum, chicken blood vine, Chuan Niu Zhi, soapberry thorn, lychee kernels, vinegar Curcuma. The pre-menstrual period is equivalent to the luteal phase of Western medicine, during which the yang is long, and the yin is fading, and patients preparing for pregnancy should tonify yin and yang, tonify the kidneys and strengthen the fetus, promote the function of the corpus luteum and nourish the fetus.

\subsection{Take care of the spleen and stomach to nourish the source of energy}

As the late menstrual period nourishes the kidney yin, the medication is too nourishing and greasy, and the drug is taken for an extended period, resulting in overburdening the spleen and stomach, leaving the spleen and stomach deficient and unsupplemented, and damaging the spleen and stomach. Professor Zhang Xiaofeng clinically often uses Chen Pi, Poria, Mu Xiang, fried Citrus aurantium, white lentils, sand seeds, coix seeds, raw hawthorn, jiao hawthorn, Shen $\mathrm{Qu}$, and chicken Beijing to strengthen the spleen and regulate qi and dampness. On the one hand, they promote the transportation of Yin nourishing drugs, and on the other hand, they take care of the spleen and stomach, which are the basis of nature and the source of biochemical blood. If the spleen and stomach are healthy and gore is abundant, the sea of blood will be plentiful, and the functions of the uterus and veins will function normally.

\subsection{Lit. examine a case and cite some examples}

Tian, 31 years old, presented to the clinic on October 21, 2019, with the chief complaint of "uncontraceptive and not pregnant for more than one year". Menstruation 7/23-25, amp: $10-11,3$ days net, the amount can, dark red colour, blood clots, mild abdominal cramps. Bring down the tone. Nashu can be, second stool tone. Red tongue, little moss. Light pulse. History of pregnancy and childbirth: G1P0, biochemical pregnancy in 2018, uterine clearance. There is a history of acute appendicitis and mycobacterial vaginitis. The first one is a woman who has a history of pregnancy, and the second one is a woman who has a history of pregnancy, and the third one is a woman who has a history of pregnancy. "Chocolate cyst on the right side." In 
September, a tubal imaging scan showed that both sides were open. Today's Yin ultrasound: endometrium $5 \mathrm{~mm}$, uterus $45 \mathrm{~mm} * 39 \mathrm{~mm} * 29 \mathrm{~mm}$, left dominant follicle $7 \mathrm{~mm} * 8 \mathrm{~mm}$, bilateral basal follicle number about 9 , right coincidental sac $14 \mathrm{~mm} * 12 \mathrm{~mm}$, diagnosis: 1 . infertility (Yin deficiency blood heat type); 2. right chocolate cyst. Prescription: Dang Gui 12g, stir-fried Paeonia lactiflora $15 \mathrm{~g}$, Sheng Di $15 \mathrm{~g}$, Dang Shen $15 \mathrm{~g}$, stirfried Atractylodis Macrocephalae $15 \mathrm{~g}$, Cuscuta sinensis $15 \mathrm{~g}$, Morinda officinalis $12 \mathrm{~g}$, Salvia miltiorrhizae $15 \mathrm{~g}$, Radix et Rhizoma heterophylla 20 g, Pericarpium cheap $10 \mathrm{~g}$, Poria cocos $15 \mathrm{~g}$, Rhizoma cyperus $10 \mathrm{~g}$, Lychee kernels $15 \mathrm{~g}$ crushed, $6 \mathrm{~g}$ Glycyrrhiza glabra, Moutan peony bark $9 \mathrm{~g}$, Chasteberry seeds $15 \mathrm{~g}$, Mo dry lotus $15 \mathrm{~g}$, stir-fried Chinese yam $15 \mathrm{~g}$, Wine Huang Jing 15 g, 5 times. Instruct the patient to take the basal body temperature and return to the clinic five days later.

October 25, 2019, Second Diagnosis. Lamp: 1011, Nahue can, second stool tone. Red tongue, little moss, delicate pulse. Lower band tone. Loose stools, nashu can. Today's egg test: endometrium $7.5 \mathrm{~mm}$, left dominant follicle $15^{*} 14 \mathrm{~mm}$. Basal body temperature (BBT): low phase. Prescription: front plus Poria $15 \mathrm{~g}$, vinegar turtle shell $20 \mathrm{~g}$ first decoction, yam easy for fried yam, 2 times. Instruct the patient to return two days later.

October 27, 2019, three diagnoses. Lamp: 10-11, nashu can, two stool tone. Red tongue, little moss, delicate pulse. The band was drawn and transparent. Today's egg test: endometrium $8 \mathrm{~mm}$, left dominant follicle $19^{*} 18 \mathrm{~mm}$. BBT: no high phase. The treatment is to warm yang and invigorate blood, eliminate symptoms and disperse knots. Prescription: danggui $12 \mathrm{~g}$, chuanxiong $10 \mathrm{~g}$, dan shen $15 \mathrm{~g}$, chicken blood vine $20 \mathrm{~g}$, astragalus $30 \mathrm{~g}$, LuLutong $12 \mathrm{~g}$, soaps or thorn $12 \mathrm{~g}$, stone see perforation $15 \mathrm{~g}$, oyster $30 \mathrm{~g}$ first decoction, cheap $10 \mathrm{~g}$, lychee kernel $15 \mathrm{~g}$ broken, vinegar trillium $12 \mathrm{~g}$, vinegar curcumin $12 \mathrm{~g}$, burning licorice $6 \mathrm{~g}$, deer horn piece $10 \mathrm{~g}$, two pay. Instruct the patient to continue to monitor the basal body temperature. Same room every other day.

October 29, 2019, four diagnoses. Lamp: 10-11. Sleep poorly, na can, two stool tone: red tongue, little moss, smooth pulse. I'm not sure if I'm going to be able to do that. The treatment is to flatten and tonify yin and yang, detoxify the liver and strengthen the spleen. Prescription: Dang Gui 12g, stir-fried Paeonia lactiflora 15g, Dang Shen 15g, stir-fried Atractylodis Macrocephalae 15g, Cuscuta Sinensis 15g, Morinda
Officinalis $12 \mathrm{~g}$, Chen Pi $10 \mathrm{~g}$, Poria cocos $15 \mathrm{~g}$, stirfried yam $15 \mathrm{~g}$, wine Huang Jing $15 \mathrm{~g}$, raw Chai $\mathrm{Hu}$ $6 \mathrm{~g}$, stir-fried wheat germ $15 \mathrm{~g}$, burning licorice $6 \mathrm{~g}$, salt eucommia $15 \mathrm{~g}$, mulberry $15 \mathrm{~g}$, continue to cut off $15 \mathrm{~g}$. Ten pay. The patient was instructed to continue monitoring basal body temperature.

Nov. 11, 2019, V. Diagnosis. Lamp: 11-10, volume ok, slightly dark, thin, clots, with mild abdominal cramps, cold abdomen. Nasu can be, second stool adjusted. Red tongue, less moss, smooth pulse. Treatment to warm and invigorate Blood. Prescription: Dang Gui 12g, Ligusticum Chuanxiong 10g, Salvia miltiorrhiza $15 \mathrm{~g}$, Chick's blood vine $20 \mathrm{~g}$, motherwort 15g, Chuan Niu Zhi 15g, Astragalus Membranaceus 30g, Chen Pi 10g, Ze Lan 15, vinegar Curcuma longa $12 \mathrm{~g}$, cumin $12 \mathrm{~g}$, burning licorice $6 \mathrm{~g}$, four pay.

After three cycles of treatment, the patient tested positive for urine HCG on January 15, 2020, and checked blood hCG and progesterone, all of which were normal.

Note: The patient had previously monitored her follicles for four menstrual cycles, all of which were small follicles ovulating. The red tongue, little moss and delicate pulse are all symptoms of yin deficiency and Blood-Heat; yin deficiency produces internal heat for a prolonged period, causing dark red and viscous menstrual flow. It's also a good idea to have a good number of people in your life who are looking for a way to make sure you're getting the best out of your money. This leads to the Kidney-Yin deficiency BloodHeat type. This is the first time the patient was seen, on the tenth day of the menstrual cycle, and the ultrasound showed that the endometrium was thin. The follicles were small, in the early stages of the late menstrual period when the Qi and blood activity was more static. It is also a good idea to use a variety of other products, such as Ligustrum lucidum and Mo dry lotus, to strengthen the yin nourishing and heat-clearing power. Angelica Sinensis, sautéed white peony, Chinese yam, wine and yellow wine tonify blood and nourish yin to nourish follicles and endometrial growth. Cuscuta Sinensis, Morinda Officinalis, tonifies the Kidney-Yang and maintains the dynamic balance of yin and yang at the end of menstruation. Chen Pi, Dang Shen, Bai Zhu, four herbs tonify Qi, strengthen the spleen, make the middle states healthy, and nourish the source of energy, and combined with Salvia divinorum, hen's blood vine, and $\mathrm{Xiang} \mathrm{Fu}$, invigorate blood to make the whole prescription tonify without stagnation. At the second 
consultation, the patient's stools were scanty and considering the patient's spleen and stomach deficiency, the yam was quickly fried to enhance the spleen's efficacy, and the poria was added to strengthen the spleen and promote diuresis to solidify the stool, adding vinegar turtle shells to improve the nourishment for yin and clear heat. At the time of the third consultation, the patient was at the end of the late menstrual period, with clear, streaky leucorrhoea, and the dominant follicle had matured, the endometrium was also available. It was about to enter the intermenstrual ovulation period. At this time, instructor Zhang Xiaofeng uses warming yang, invigorating blood, dispersing knots and dissipating symptoms to induce follicle discharge, Danshen, hen's blood vine, Angelica Sinensis, Chuanxiong, nourishing Blood and invigorating Blood. Astragalus tonifies Qi and aids Blood movement. Lu Tong, soap thorn, lychee kernel, oyster, trillium, Curcuma, and stone see perforated herbs disperse knots and subdue symptoms. Chen Pi strengthens the spleen and protects the spleen and stomach. The combined use of these herbs helped to break up the egg and cause the egg to be discharged. The patient's dominant follicle had been removed by the time of the fourth consultation, and the luteinizing function was promoted by tonifying yin and yang, sparing the liver and strengthening the spleen to nourish the fetus. Dang Gui, fried white atractylodes, tonifies qi and strengthens the spleen, while Cuscuta Sinensis, Morinda Officinalis, Eucommiae, Continued Flare, and Mulberry sorrel tonify yin and yang. Chen Pi, Fu Shen, strengthens the spleen and calms the spirit; Chai $\mathrm{Hu}$, fried wheat germ, evacuates the liver and regulates Qi. When the five cherished, the patient menstrual flow is not pregnant, but the gentle menstrual cycle is regular, Zhang Xiaofeng tutor believes that the primary menstrual "leakage" of the work, at this time it is appropriate to clear the uterus, to facilitate the late menstrual "hide". So above all for the warmth and blood medicine, lead blood downward. So treatment, the third menstrual cycle patient pregnancy positive, continue to fertility preservation treatment.

\section{Concluding remarks}

Zhang Xiaofeng has been practising medicine for more than 30 years and believes that the pathogenesis of small follicular, ovulatory infertility is involved, which is often accompanied by menstruation. The clinical treatment is based on traditional Chinese medicine artificial cycle therapy, sequential procedure, emphasis on nourishing kidney yin, coupled with ultrasound monitoring of the synchronous development of the follicle and endometrium, the clinical treatment effect is considerable.

\section{References}

[1] The clinical significance of ultrasound monitoring of follicular development for unexplained infertility[J]. South China University Journal of Medicine, 2005, 33(3): 382-383.

[2] Luo LL. Infertility [M]. Beijing: People's Health Press, 2009: 202-203. 